\title{
Topical analgesia for superficial corneal injuries
}

\author{
A K Brahma, S Shah, V F Hillier, D McLeod, T Sabala, A Brown, J Marsden
}

\begin{abstract}
Objective-To assess the analgesic effects of a topical non-steroidal anti-inflammatory agent, flurbiprofen $0.03 \%$, during healing after superficial corneal injuries. Methods-401 patients treated for corneal abrasion in a five month period were randomly allocated to one of four treatment groups: polyvinyl alcohol alone (control), homatropine $2 \%$, flurbiprofen $0.03 \%$, or homatropine $2 \%$ followed by flurbiprofen $0 \cdot 03 \%$. Treatments were given for $48 \mathrm{~h}$. Ocular pain was recorded on a visual analogue scale by the patients over the first $24 \mathrm{~h}$, and use of oral analgesics was also recorded. Usable responses were received from 224 patients $(55 \cdot 8 \%)$.

Results-Patients treated with flurbiprofen had significantly lower pain scores for the $24 \mathrm{~h}$ duration of the study than controls $(P<0.05)$.

Conclusions-Flurbiprofen eye drops provide more effective pain relief than traditional treatments for superficial corneal injuries.
\end{abstract}

(f Accid Emerg Med 1996;13:186-188)

Key terms: corneal abrasions; corneal foreign bodies; flurbiprofen; non-steroidal anti-inflammatory agents; topical analgesia

Superficial corneal injuries, with abrasions or embedded foreign bodies, are the most frequent ophthalmic presentations to an accident and emergency (A\&E) department. ${ }^{1}$ The associated discomfort is often severe and can last for many hours, if not days. Traditionally, instillation of a topical cycloplegic, for example homatropine $2 \%$, and an antibiotic, together with the application of an eye pad, has been used to ease pain and promote healing.

Prostaglandins and other metabolites of arachidonic acid play a major role in pain sensation by altering the sensitivity of the nociceptive neurones. ${ }^{2}$ Non-steroidal antiinflammatory drugs (NSAIDs) are commonly used systemically as analgesics in a variety of painful conditions. ${ }^{3}$ Their effect is to inhibit the enzyme cyclooxygenase and thus to decrease the synthesis of prostaglandins. ${ }^{4}$ The aim of this study was to assess the analgesic effect of topically instilled flurbiprofen $0.03 \%$, an NSAID, on the level of pain associated with corneal abrasions and foreign bodies.

\section{Methods}

Medical ethics committee approval for this randomised clinical study was obtained. Between August 1993 and December 1993 all patients with corneal abrasions and foreign bodies attending the emergency eye centre at
Manchester Royal Eye Hospital (and who had not received any previous treatment) were asked to volunteer for the study. All patients gave informed consent. The following were excluded from study: patients under the age of 16 years, pregnant women, and those with a history of herpes simplex keratitis or a known hypersensitivity to NSAIDs.

A pilot study was undertaken before the start of the trial: 10 patients with superficial corneal injuries received flurbiprofen $0.03 \%$ eye drops four times daily; the vehicle for this NSAID was polyvinyl alcohol $1 \cdot 4 \%$ (Liquifilm tears, Allergan). The patients were reviewed until the injury had healed in order to assess any adverse effects of the topical NSAID. There were no any side effects except a transient burning sensation on instillation of the eye drops. In view of this, and our normal practice of "no routine follow up" of superficial corneal injuries, we decided to assess the symptomatic outcome of treatment in a large series using a postal questionnaire. Statistical advice taken before the start of the randomised clinical study suggested that at least 200 subjects would be required to detect a significant difference between the treatment and control groups at the $P=0.05$ level. Since postal questionnaires usually have a return rate of approximately $50 \%$, it was planned to recruit 400 patients.

Patients were consecutively allocated at random to one of four treatment groups (table 1). Groups 1 and 2 were the "control" and "normal practice" groups, and groups 3 and 4 were the NSAID treatment groups. All patients received an initial dose of chloramphenicol eye ointment and an eye pad; they were asked to remove their eye pad after $6 \mathrm{~h}$ and to instil chloramphenicol $1 \%$ ointment four times daily for $5 \mathrm{~d}$. All ophthalmic preparations were preservative-free. The size of the corneal abrasion and site of the foreign body was noted in each case.

Before any treatment the patients were asked to assess their ocular pain on a $10 \mathrm{~cm}$ linear visual analogue scale (VAS). The linear scale was marked 0 - indicating no pain, and $10-$ indicating the worst pain ever experienced;

\section{Table 1 Treatment groups}

Group 1 Polyvinyl alcohol $1.4 \%$ drops (Liquifilm Tears) four times daily for $\mathbf{4 8}$ hours: the control group.

Group 2 Stat instillation of homatropine $2 \%$ drops at presentation only: normal practice group.

Group 3 Flurbiprofen $0.03 \%$ drops four times daily for 48 hours: the first treatment group.

Group 4 Stat instillation of homatropine $2 \%$ drops at presentation only, and flurbiprofen $0.03 \%$ drops four times daily for 48 hours: the second treatment group.

All patients received chloramphenicol eye ointment to be used \begin{tabular}{l} 
four times daily for five days. \\
\hline
\end{tabular}

V F Hillier 
Table 2 Demographic data and initial pain scores by treatment group

\begin{tabular}{|c|c|c|c|c|}
\hline & Group 1 & Group 2 & Group 3 & Group 4 \\
\hline \multicolumn{5}{|c|}{ Patients entering the study $(n=401)$} \\
\hline Male & 81 & 84 & 74 & 84 \\
\hline Female & 19 & 16 & & 17 \\
\hline Mean age (years) & $35 \cdot 1$ & $33 \cdot 3$ & $32 \cdot 7$ & $33 \cdot 8$ \\
\hline \multicolumn{5}{|c|}{ Patients returning the completed questionnaire $(n=224)$} \\
\hline Male & 48 & 51 & 43 & 45 \\
\hline Female & 9 & 7 & 14 & 7 \\
\hline Mean age (years) & $35 \cdot 7$ & $32 \cdot 8$ & $32 \cdot 5$ & $35 \cdot 7$ \\
\hline Initial pain score $(\mathrm{cm})$ & $5 \cdot 6$ & $5 \cdot 5$ & $5 \cdot 5$ & $5 \cdot 5$ \\
\hline \multicolumn{5}{|c|}{ Patients failing to return completed questionnaire $(n=177)$} \\
\hline Male & 33 & 33 & 31 & 39 \\
\hline Female & 10 & 9 & 12 & 10 \\
\hline Mean age (years) & $34 \cdot 4$ & $34 \cdot 0$ & $33 \cdot 0$ & $32 \cdot 5$ \\
\hline Initial pain score $(\mathrm{cm})$ & $5 \cdot 8$ & $5 \cdot 7$ & $5 \cdot 8$ & $5 \cdot 7$ \\
\hline
\end{tabular}

there were no gradations or markings between 0 and 10. At six hourly intervals for $24 \mathrm{~h}$ thereafter, patients were asked to mark their level of pain on the VAS. If a $6 \mathrm{~h}$ time point occurred during sleep patients were asked to complete the appropriate pain score on waking. Oral analgesics were permitted: in the patient information sheet paracetamol $1 \mathrm{~g}$ six hourly was recommended. The use of oral analgesia (whether paracetamol or otherwise) was simply recorded with a yes/no answer on the questionnaire. In addition sleep was graded normal or disturbed, and patients were asked to record whether or not they had time off work because of their eye injury.

The patients were asked to return their completed questionnaire and VAS sheet by prepaid post. Those with residual rust rings or abrasions involving more than one third of the corneal surface area were reviewed in the emergency eye centre. The pain score indicated on each VAS was measured using a ruler with millimetre markings.

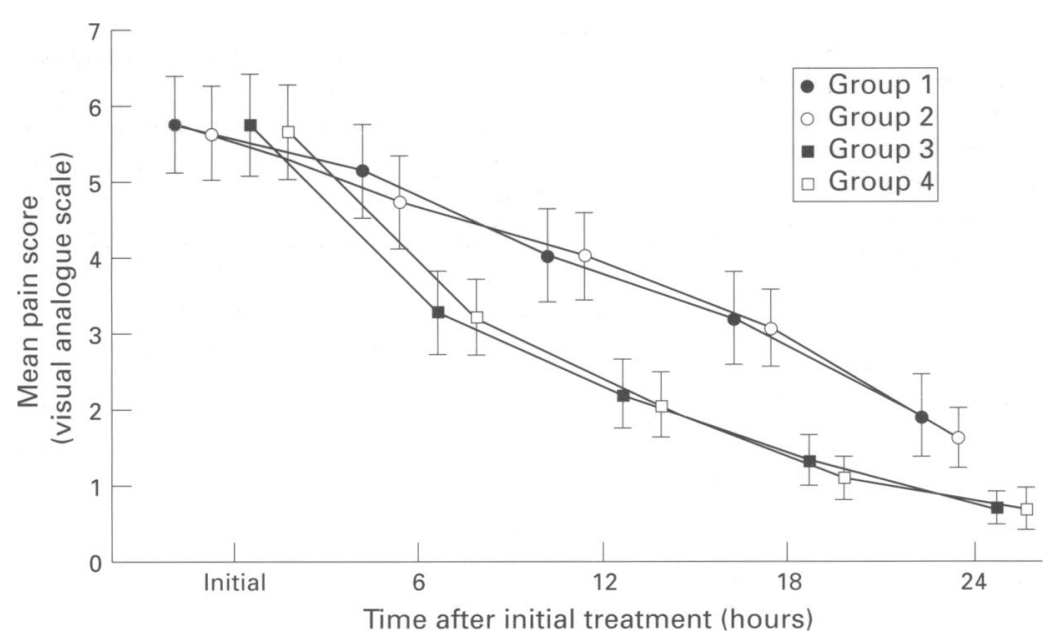

Variation of mean pain score in treatment groups with time after initial treatment. Error bars indicate $95 \%$ confidence intervals.

Table 3 Use of oral analgesia, sleep disturbance, and time off work by treatment group

\begin{tabular}{llllllll}
\hline & & Group 1 & Group 2 & Group 3 & Group 4 & $\chi^{2}$ & Pvalue \\
\hline Oral analgesia & Yes & 29 & 37 & 13 & 16 & 24.38 & $<0.01$ \\
& No & 28 & 21 & 44 & 36 & & \\
Sleep disturbance & Yes & 22 & 24 & 10 & 12 & 31 & $<0.01$ \\
& No & 35 & 34 & 47 & 40 & & \\
Time off work & Yes & 24 & 28 & 8 & 10 & $21 \cdot 1$ & $<0.01$ \\
& No & 33 & 30 & 49 & 42 & & \\
\hline
\end{tabular}

On cross tabulation, groups 3 and 4 are significantly different from groups 1 and 2 . $\chi^{2}$ Analysis was performed on sex distribution, use of oral analgesia, sleep disturbance, and time off work. Kruskal-Wallis one way analysis of variance (ANOVA) was used to analyse the pain scores at each time point between the treatment groups. Repeated measures of analysis of variance (MANOVA) was used to compare the four groups over the 24 $\mathrm{h}$ period. Student's $t$ test and $\chi^{2}$ test were used to analyse data on age, sex distribution, and initial pain score between those who completed and those who did not complete the study.

\section{Results}

A total of 401 patients volunteered to participate in the study: 202 and 199 patients had corneal foreign bodies and abrasions respectively. Of these patients, 224 returned appropriately completed questionnaires $(55.8 \%$ reply rate); the number of patients in each treatment group is shown in table 2 . There was no statistically significant difference between those not completing and those completing the study in the following variables: age $(P>0 \cdot 1)$; sex $(\mathbf{P}>0 \cdot 1)$; and initial pain score $(\mathbf{P}>0 \cdot 1)$. The initial pain scores (pretreatment) were normally distributed between all groups (KolmogrovSmirnov goodness of fit test mean: $5 \cdot 709$, SD $2 \cdot 39, P=0 \cdot 13$ ), and there was no significant difference between the groups in respect of age and sex.

Of those patients returning appropriately completed questionnaires and VAS sheets, the patients receiving flurbiprofen (groups 3 and 4) had reduced pain scores $(P<0.05$, MANOVA) compared to the control and normal practice groups (groups 1 and 2) during the first $24 \mathrm{~h}$ after injury and initial treatment (figure). At each time point, the groups receiving flurbiprofen (groups 3 and 4 ) had reduced pain scores compared to the control and normal practice groups (groups 1 and 2$)(P<0.05$, ANOVA). There were no significant differences in the pain scores between groups 3 and 4 for each time point and over the $24 \mathrm{~h}$ period ( $P>0.05$, ANOVA; $\mathbf{P}>0.05$ MANOVA); this also applied to the control and normal practice groups (groups 1 and 2).

Patients receiving flurbiprofen (groups 3 and 4) took less oral analgesia than the controls (groups 1 and 2) $(P<0.01)$; in groups 3 and 4 more patients had normal sleep than in groups 1 and $2(P<0.05)$; in groups 3 and 4 fewer took time off work $(P<0.01)$ (table 3$)$. No complications were encountered during the study: 22 patients with corneal abrasions and 26 with corneal foreign bodies were reviewed for side effects; of these 46 patients $(20.5 \%$ of those completing the study), 21 were in groups 3 and 4 . Some patients who initially received both homatropine $2 \%$ and flurbiprofen $0.03 \%$ noted a burning sensation lasting a few seconds.

\section{Discussion}

We have shown that, compared with traditional practices for the management of superficial corneal injuries, flurbiprofen $0.03 \%$ applied topically provides significantly improved analgesia. 
Table 4 Guidance notes on superficial corneal injuries

1 Assessment

(a) History of injury (cause, duration, loss of vision, pain workplace related, use of powertools etc, eye protection, exclude intraocular foreign body)

(b) Visual acuity for each eye (with and without glasses). If the patient does not have his glasses, assess the visual acuity with a pinhole.

(c) Ocular examination with a magnifying loupe or a slit lamp. Fluorscein staining of the cornea. If there is a foreign body remove it.

2 Treatment

(a) Removal of foreign body

b) Prophylactic topical antibiotic (this must be continued if the foreign body/rust ring persists).

(c) Topical flurbiprofen if painful.

(d) Cycloplegia in the presence of intraocular inflammation.

3 Follow up only if

(a) Intraocular inflammation (refer to an ophthalmogist)

b) Patient to return if pain or vision worsening or still painful after 48 hours.

Seek the advice of an ophthalmologist if in doubt!

The cornea is particularly suited for topical analgesic preparations as it is readily accessible. It is one of the most sensitive tissues in the body, with the highest density of sensory neurones per $\mathrm{mm}^{2}$ just below the epithelium (four to five times greater than in the finger pads). ${ }^{5}$ All varieties of stimulation (mechanical, chemical, or thermal) are perceived as pain. ${ }^{6}$ This nociceptive sensitivity is essential for the integrity of the cornea, preventing further damage. Epithelial loss leads to exposure of the subepithelial plexus of nerve endings; they are then stimulated by the mechanical action of the eyelids opening and closing and by noxious chemicals in the tears. ${ }^{5}$ The exposed nerve endings are sensitised by metabolites of arachidonic acid. NSAIDs are effective in reducing the synthesis of these metabolites, leading to alteration of the pain threshold. ${ }^{7}$ Sodium diclofenac, another NSAID, significantly lowered corneal sensitivity in normal eyes $^{8}$ and reduced discomfort, pain, and inflammation following photorefractive keratoplasty ${ }^{9}$; this corroborates our finding.

The rate of corneal epithelialisation was not assessed in this study, but the effect of a variety of topical NSAIDs (including flurbiprofen) on corneal wound healing has been well documented previously. ${ }^{10-12}$ None of the published studies reported any significant adverse effects, and neither patients in our pilot study nor those returning for removal of residual rust rings or review of large corneal abrasions showed any complications attributable to the topical NSAID.

In conclusion, flurbiprofen eye drops provide effective and significant pain relief compared to the traditional treatments for superficial corneal injuries. All patients attending a general $A \& E$ department or a dedicated eye casualty department with superficial corneal injuries should be assessed and treated appropriately (table 4 ; guidance notes).

We would like to thank: the Nursing staff of the Royal Eye Hospital, Manchester for their advice, support, and recruitment of patients; Allergan, UK for providing the eye medications; and Mr K Mackway-Jones, Consultant in Accident and Emergency Medicine at Manchester Royal Infirmary, for reviewing the manuscript. None of the authors has any financial interest in Allergan Therapeutics.

1 Vernon S. Analysis of all new cases seen in a busy regional centre ophthalmic casualty department during a 24 week centre ophthalmic casualty department

2 Davies P, Bailey PJ, Goldeberg MM, Ford-Hutchinson AW. The role of arachidonic acid oxygenation products in pain and inflammation. Annu Rev Immunol 1984;2:335-57.

3 Kantor TG. Use of diclofenac in analgesia. Am $\mathcal{F}$ Med $1986 ; 80$ (suppl 4B):64-9.

4 Vane JR. Inhibition of prostaglandin synthesis as a mechanism of action for aspirin-like drugs. Nature New Biol 1971;231:232-9.

5 Rozsa AJ, Beuerman RW. Density and organisation of free nerve endings in the corneal epithelium of rabbits. Pain 1982;14:105-20.

6 Tanelian DL, Beuerman RW. Responses of rabbit corneal nociceptors to mechanical and thermal stimulation. Exp Neurol 1984;84:165-78.

7 Ferriera SH. Prostaglandins and hyperalgesia, and the control of inflammatory pain. In: Bonta IL, Bray MA, Parnham MJ, eds. Handbook of inflammation, vol 5, The pharmacology of inflammation. NewYork: Elsevier, 1985: pharmacolog.

8 Szereny $\mathrm{K}$, Sorken $\mathrm{K}$, Garbus J, Lee M, McDonnel P. Decrease in normal human corneal sensitivity with topical diclofenac sodium. Am $f$ Ophthalmol 1994;118:312-5.

9 Herschel MK, McDonald MB, Ahmed S, Klyce RJ, Varnell RJ, Thompson HW. Voltaren for treatment of Varnell RJ, Thompson HW. Voltaren for treatment of
discomfort after excimer ablation [ARVO abstracts]. discomfort after excimer ablation [ARV
Invest Ophthalmol Vis Sci (suppl) 1993:893.

10 McGarey BE, Napalkav JA, Pippen PA, Reaves TA. Corneal wound healing strength with topical anti-inflammatory drugs [ARVO abstracts]. Invest Ophthalmol Vis Sci 1993: 1321 .

1 Srinivasan BD, Kulkarni PS. The effect of steroidal and non steroidal anti-inflammatory agents on corneal reepithelialisation. Invest Ophthalmol Vis Sci 1981;20: 688-91.

12 Hersh A, Rice BA, Baer JC, Wells PA, Lynch E, McGuigan LJB, et al. Topical nonsteroidal agents and corneal wound healing. Arch Ophthamol 1990;108: $577-83$ 